\title{
Surveillance of Drying Processes of Forage Layers in Silos
}

\author{
Dr. rer. nat. Astrid Stacheter, M. Eng. David Becker \\ Institute of Sensor and Actuator Technology, Coburg University of Applied Science and Arts, \\ Am Hofbräuhaus 1b, Coburg, Germany \\ A. Stacheter@isat-coburg.de
}

\begin{abstract}
Organic particle depositions at the inner side of tower silo walls present health risks for animals and humans, creating a habitat for mycotoxin producing molds. Mold proliferation and mycotoxin production in forage substrates are influenced by abiotic factors such as the water content. Until now, a non-invasive detection method of forage depositions in silos has not been available, making it difficult to remove them accurately timed to prevent of fungal infestation. In the current study a Lamb wave based acoustical sensor principle was developed in order to detect wet organic depositions at the inner wall of steel silos. It was shown that forage layers on the propagation track of Lamb waves impact on propagation velocity and maximum amplitude of Lamb waves based on mass loading and mode conversion. The maximum amplitude and the propagation time were much more dependent on the humidity of the forage layer than on layer thickness. Therefore the sensor principle was suitable to detect important key factors of mold infestation in forage silos.
\end{abstract}

Key words: Acoustical Sensor, Lamb Wave, Forage Layer, Non Invasive Detection, Silo

\section{Introduction}

At the beginning of the manufactory chain of animal products there is the forage. Forage has to be stored hygienically in order to guarantee high forage quality and thus animal and human health. Under improper storage conditions such as a relative humidity value above $70 \%$ the growth of spoilage molds is favored. Spoilage molds can cause allergic reactions, for example farmer's lung or asthma [1]. Furthermore they can produce poisonous mycotoxins like Aflatoxin $B_{1}$ [2], which is classified as a potential animal carcinogen [3]. A main risk factor for forage deterioration is the formation of moist forage layers at the inner silowall as a result of water condensation and filling processes of the silo. If not removed in time, moist forage layers provide optimal growth conditions for molds and, falling of the silo wall, they lead to forage contamination [1]. A sensor for wet forage layers holds a key to optimal storage management enabling countermeasures such as silo cleaning at the right time. However, a non-invasive technique to detect moist forage layers on silo walls is currently not available. Various layer detection technologies such as electrochemical or optical methods are applied in industry but these sensors have the disadvantage that they have to be in direct contact with the layer $[4,5]$. That means that in the case of a closed system the sensor apparatus has to be installed permanently inside the system leading to difficulties for example by hindering cleaning processes. Lamb waves offer the possibility to detect layers noninvasively [6]. It was shown previously that they can be applied to monitor erosion and corrosion of pipes [7] and to detect baked food products [8]. Furthermore it is known that the propagation of the Lamb wave along a solidliquid interface is influenced by the liquid [9]. The objective of this study was to investigate if Lamb waves can also be applied to detect wet forage layers in steel silos and to monitor drying processes.

\section{Methods}

The measurement setup consisted of a steel plate with a thickness of $1.5 \mathrm{~mm}$ which was equipped with two single phase transducers per propagation track, one transmitter and one receiver transducer (Fig. 1). A single phase transducer consists of a piezoelectric lead zirconate titanate block with a metallized comb pattern on one side and a completely metallized surface on the other side. The pitch of the fingers of the comb structure determines the wavelength of the excited wave [6]. By using a single phase transducer with a finger pitch of 3 $\mathrm{mm}$ Lamb waves were generated, since the 
wavelength was larger than the thickness of the steel plate. The signal was generated with a function generator (33210A, Agilent, Santa Clara, US) by sinusoidal $5 \times$ burst excitation with an amplitude of $20 \mathrm{Vpp}$. The signal at the receiver transducer was displayed with a digital storage oscilloscope (WaveRunner 604Zi, LeCroy, Chestnut Rich, US).

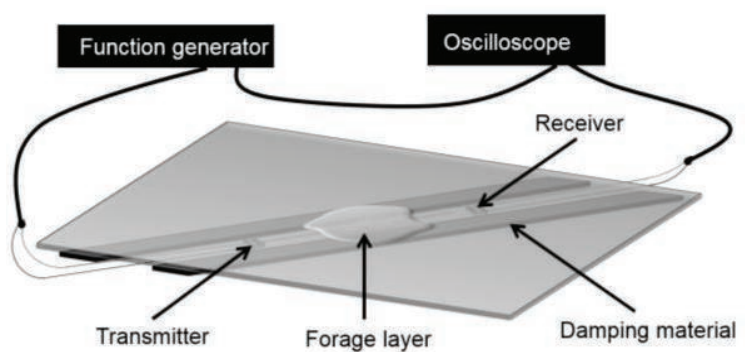

Fig. 1. Laboratory measurement concept. The transmitter as well as the receiver were single phase transducers and glued onto one side of the steel plate. The forage layer consisting of a water-forage mixture was applied onto the steel plate surface on the opposite side of the transducers.

The transmission time of the wave was determined at a predefined zero crossing of the receiver signal and the amplitude was calculated by peak to peak measurement. In order to simulate wet forage layers animal forage was sieved, mixed with water and applied onto the steel surface in form of a defined area $(6 \mathrm{~cm} \times 6 \mathrm{~cm})$ and in defined thicknesses ( $1 \mathrm{~mm}, 2 \mathrm{~mm}, 3 \mathrm{~mm}, 6 \mathrm{~mm}$ ). The temperature of the setup was kept constant by enclosing it in a temperature chamber.

Since Lamb waves are dispersive waves, the product of excitation frequency and plate thickness determines the phase velocity for a given mode of the Lamb wave [10]. In order to excite $A_{0}$ modes of Lamb waves an excitation frequency of $820 \mathrm{kHz}$ was applied as simulations with the software vallen dispersion indicated that at this frequency the Ao mode and So mode can be easily distinguished by their different phase velocities. A scanning Laser Doppler Vibrometer (LDV) (PSV-400, Polytec, Hörsching, AT) was used in order to determine the wave propagation on the steel plate and the vibration behavior of the transmitter at these excitation parameters.

\section{Results}

The LDV measurement indicated that Lamb waves were excited by a transmitter and propagated through a forage layer on the plate surface from the right side (Fig 2 A) to the left side of the steel plate (Fig $2 \mathrm{~B}$ ). The wavelength $\lambda$ of the excited mode was $2.9 \mathrm{~mm}$ (Fig. $2 \mathrm{~A}$ ). The phase velocity calculated from the wavelength and the excitation frequency via wave equation was $2380 \mathrm{~m} / \mathrm{s}$ and was in good agreement with theoretical velocity values of the Ao mode of the dispersion curve $(2400 \mathrm{~m} / \mathrm{s}$, Fig 3). Conclusively LDV measurement verified that the measurement setup was suitable to excite and detect $A_{0}$ modes of Lamb waves on steel.

A

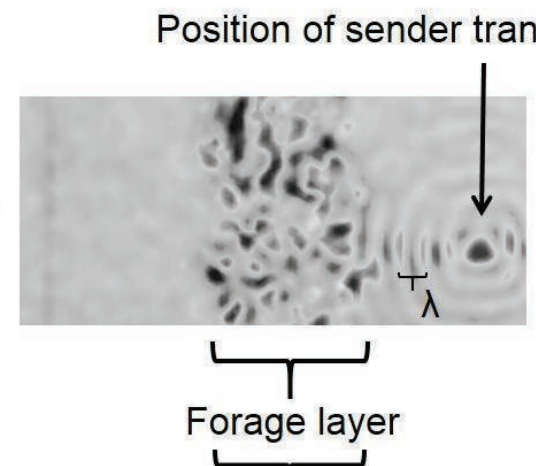

B

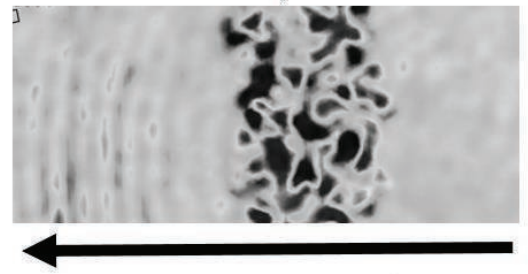

Propagation direction

Fig. 2. Results of the LDV measurements. The propagation of the Lamb wave was recorded at $7 \mu \mathrm{s}$ (A) and $19 \mu \mathrm{s}$ (B) after excitation.

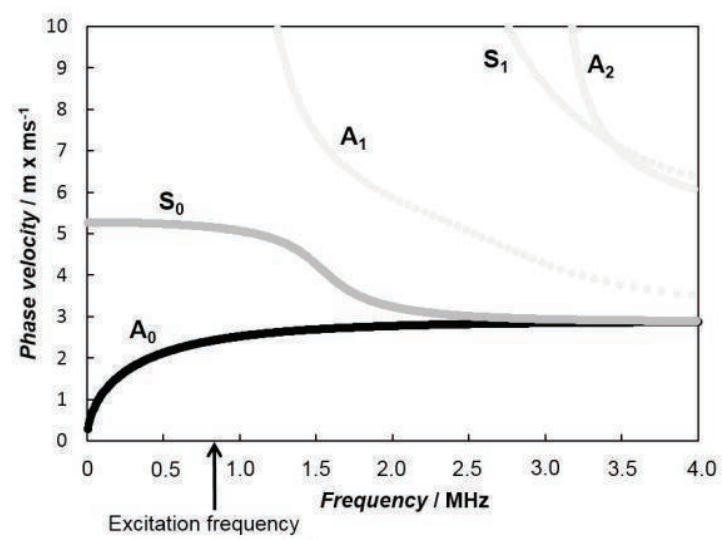

Fig. 3. Dispersion curve calculated for a $1.5 \mathrm{~mm}$ thick steel plate. At an excitation frequency of $0.82 \mathrm{MHz}$ the theoretical phase velocity of the $A_{0}$ mode is $2400 \mathrm{~m} / \mathrm{s}$.

The application of a forage layer onto the steel plate changed the signal. The amplitude decreased from $0.22 \mathrm{~V}$ without layer to $0.02 \mathrm{~V}$ after application of a $1 \mathrm{~mm}, 2 \mathrm{~mm}$ or $3 \mathrm{~mm}$ thick wet forage layer (Fig. 4). At the same time the propagation time increased by at least $0.6 \mu \mathrm{s}$ (Fig. 5), which can be easily detected by the electronic equipment. During the course of the experiment the forage layer dried and subsequently amplitude as well as propagation 
time returned to the starting level. The following relation was observed: the starting level of amplitude and propagation time was reached the earlier, the thinner the applied layer (Fig. 4, Fig. 5).

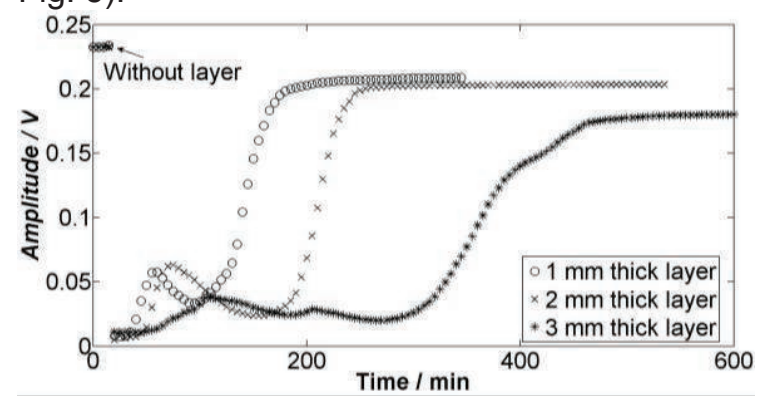

Fig. 4. Impact of wet forage depositions on the amplitude of the signal measured over drying time. The forage layer consisted of a 1:1 water forage mixture and was applied $30 \mathrm{~min}$ after measurement start. The increase of the amplitude over time is based on drying processes of the forage layer. The distance between transmitter and receiver was 10 $\mathrm{cm}$, the temperature was $50^{\circ} \mathrm{C}$.

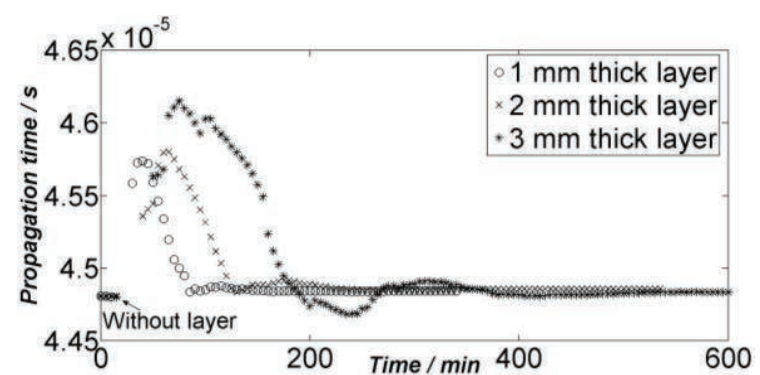

Fig. 5. Impact of wet forage depositions on the propagation time of the wave measured over drying time. The forage layer consisted of a 1:1 water forage mixture and was applied $30 \mathrm{~min}$ after measurement start. The decrease of the propagation time is based on drying processes of the forage layer. The distance between transmitter and receiver was $10 \mathrm{~cm}$, the temperature was $50^{\circ} \mathrm{C}$.

Further experiments also revealed that the water content of the forage layer influences the signal amplitude and transmission time. If $1 \mathrm{~mm}$ thick water forage layer with a mixing proportion of 0.8 to 1 was applied to the steel surface the amplitude decreased by $0.05 \mathrm{~V}$ compared to the amplitude of the signal without layer. Concomitantly transmission time increased by about $0.06 \mu \mathrm{s}$. If the proportion of the water forage layer was 1 to 0.8 the amplitude decreased by $0.10 \mathrm{~V}$ compared to the signal without layer and propagation time increased by $0.23 \mu \mathrm{s}$ (Fig. 6, Fig. 7). The effect of the layer thickness on amplitude as well as on transmission time was low compared to the effect of the water content. Water drops that were applied onto the surface in order to simulate water condensation on the silo wall had a less dominant effect on the signal (Fig. 6, Fig. 7).

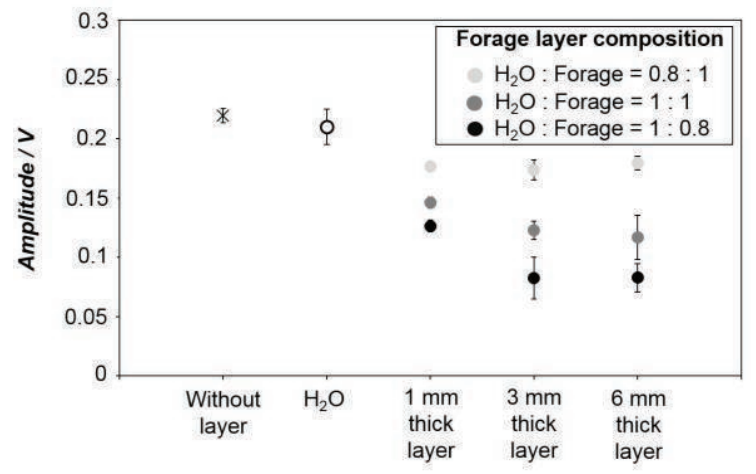

Fig. 6. Relation of signal amplitude and forage layer thickness and water content. The mean values of 3 replicates inclusive standard deviations are presented. The abbreviation $\mathrm{H}_{2} \mathrm{O}$ stands for water drops on the steel surface.

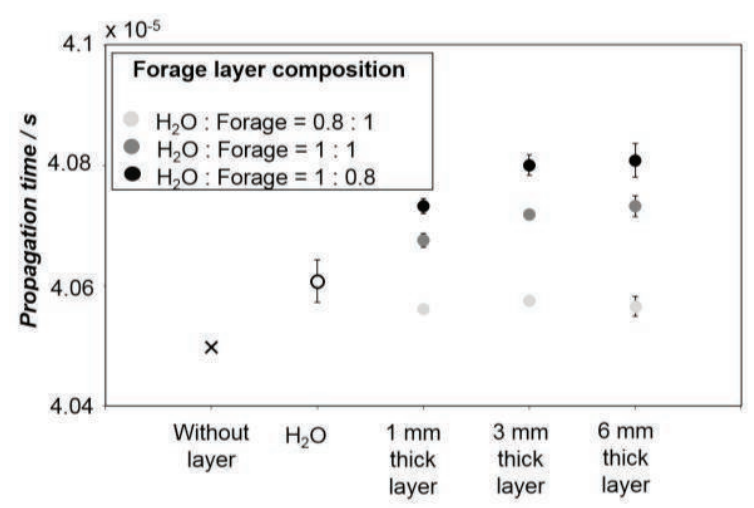

Fig. 7. Relation of signal propagation time and forage layer thickness and water content. The mean values of 3 replicates inclusive standard deviations are presented. The abbreviation $\mathrm{H}_{2} \mathrm{O}$ stands for water drops on the steel surface.

\section{Discussion and Conclusion}

Laboratory experiments showed that $A_{0}$ mode Lamb waves are suited to detect wet forage layers on steel and to monitor drying processes. Two effects that are presumably responsible for amplitude damping and reduction of the propagation velocity are mode conversion, attenuation and retardation. A high water content of the layer is the basis for a great effect of mode conversion. As the wave velocity in steel is smaller than the wave velocity in water $(1500 \mathrm{~m} / \mathrm{s})$ part of the wave energy might be refracted into the water content of the moist forage layer as longitudinal compressional wave leading to wave attenuation [9]. The sensor principle has the potential to be applied in other industrial sectors like the food industry.

\section{Acknowledgements}

This work was part of the project SenSilo and supported by the BMWi.

\section{References}

[1] J.T. Mills, Canadian Government Publishing Centre. 12-13 (1989);1823E 
[2] M. Streit, G. Schatzmayr, P. Tassis, E. Tzika, D. Marin, I. Taranu, C. Tabuc, A., Nicolau, I. Aprodu, O. Puel, I. P. Oswald, Current Situation of Mycotoxin Contamination and Co-occurence in Animal Feed - Focus on Europe, Toxins 4, 788809 (2012); doi: 10.3390/toxins4100788

[3] M. J. Sweeney, A. D. W. Dobson, Mycotoxin Production by Aspergillus, Fusarium and Penicillium Species, International Journal of Food Microbiology 43, 141- 158 (1998); doi: 10.1016/S0168-1605(98)00112-3

[4] P. M. Withers, Ultrasonic, Acoustic and Optical Techniques for the Non-Invasive Detection of Fouling in Food Processing Equipment, Trends in Food Science \& Technology 7, 293- 298 (1996); doi: 10.1016/0924-2244(96)10031-5

[5] H.C. Flemming, Role and levels of real-time monitoring for successful anti-fouling strategies an overview, Water Science and Technology 47, 1- 8 (2003)

[6] M. Schmitt, K. Schmidt, S. Olfert, J. Rautenberg, G. Lindner, B. Henning, L. M. Reindl, Detection of Coatings within Liquid-Filled Tubes and Containers by Mode Conversion of Leaky Lamb Waves, Journal of Sensors and Sensor Systems 2, 73- 84 (2013); doi: 10.2194/jsss-2-73-2013

[7] J. Pei, M. I. Yousuf, F. L. Degertekin, B.V. Honein, B. T. Khuri-Yakub, Lamb Wave Tomography and Its Application in Pipe Erosion/Corrosion Monitoring, Res Nondestr Eval 8, 189- 197 (1996) doi: 10.1007/BF02433949

[8] K.R. Lohr, J. L. Rose, Ultrasonic guided wave and acoustic impact methods for pipe fouling detection, Journal of Food Engineering 56, 315324 (2003); doi: 10.1016/S0260-8774(02)001565

[9] G. Lindner, Sensors and Actuators Based on Surface Acoustic Waves Propagation Along Solid-Liquid Interfaces. Journal of Physics D: Applied Physics, 41, 123002 (2008) doi: 10.1088/0022-3727/41/12/123002

[10] I.A. Viktorov, Rayleigh and Lamb Waves, Physical Theory and Applications, Academic Press, New York, 1967 